\title{
Civilisations
}

Revue internationale d'anthropologie et de sciences

humaines

$41 \mid 1993$

Mélanges Pierre Salmon II

\section{Le mouvement géographique, un journal et un géographe au service de la colonisation du Congo}

\section{Henri Nicolai}

\section{(apenEdition \\ Journals}

Édition électronique

URL : http://journals.openedition.org/civilisations/1710

DOI : 10.4000/civilisations. 1710

ISSN : 2032-0442

\section{Éditeur}

Institut de sociologie de l'Université Libre de Bruxelles

\section{Édition imprimée}

Date de publication : 1 septembre 1993

Pagination : 257-277

ISBN : 2-87263-094-5

ISSN : 0009-8140

\section{Référence électronique}

Henri Nicolai, « Le mouvement géographique, un journal et un géographe au service de la colonisation du Congo », Civilisations [En ligne], 41 | 1993, mis en ligne le 29 juillet 2009, consulté le 01 mai 2019. URL : http://journals.openedition.org/civilisations/1710 ; DOI : 10.4000/civilisations.1710

Ce document a été généré automatiquement le 1 mai 2019.

(c) Tous droits réservés 


\title{
Le mouvement géographique, un journal et un géographe au service de la colonisation du Congo
}

\author{
Henri Nicolai
}

1 Le premier numéro du Mouvement Géographique est publié à Bruxelles le 6 avril 1884 . Depuis près de huit ans, deux sociétés de géographie ont été fondées en Belgique, à Bruxelles et à Anvers et, après quelques mois, ont sorti leurs premiers bulletins. Le Mouvement Géographique se distingue de ceux-ci d'abord parce qu'il est un véritable journal et ensuite parce qu'il a un objectif plus précis.

\section{Les géographes belges et la colonisation}

2 Les sociétés de géographie naissent en 1876, au moment où Léopold II réunit à Bruxelles une Conférence Géographique, qui sera l'amorce de son entreprise africaine. Elles ont contribué à familiariser à l'idée coloniale une partie de l'opinion publique belge, jusqu'alors très réticente devant toute aventure de ce type ${ }^{1}$.

Cependant leurs membres fondateurs avaient mis quelque temps à retenir officiellement la promotion de l'idée coloniale parmi leurs préoccupations. En rédigeant leurs statuts et en définissant leurs objectifs, ils évitèrent même, pour la société de Bruxelles par exemple, toute référence à un encouragement en matière de colonisation, que l'un d'eux avait pourtant inscrite dans le texte initial. Et cela très curieusement parce qu'ils craignaient de se trouver privés du soutien des autorités belges dont ils connaissaient l'hostilité à de telles initiatives. Ils remplacèrent dans le texte l'expression "colonisation" par le terme "émigration"2. Mais Léopold II ayant couvert ses activités africaines d'un manteau humanitaire et scientifique, les sociétés de géographie n'hésitèrent pas à lui apporter leur appui. En 1884, on commence donc à s'habituer à l'idée que des Belges sont en train de s'occuper du Congo et, même si cela rencontre encore beaucoup de résistance parmi les hommes politiques, on sait que la Belgique, indirectement tout au moins, va se trouver concernée par une opération coloniale. 
4 Les sociétés de géographie ont donc rendu compte fidèlement de l'activité de l'Association Internationale Africaine puis rapporté soigneusement les progrès de l'Etat Indépendant du Congo mais elles ne feront pas, de l'action coloniale, leur matière spécifique. Leurs bulletins s'occuperont aussi de la géographie de la Belgique, de celle de l'Europe et des autres parties du monde et s'intéresseront par exemple aux terres encore à découvrir dans les régions antarctiques.

\section{Un objectif spécifique}

5 Au contraire, le Mouvement Géographique aura comme activité esentielle la promotion de l'action coloniale belge en Afrique. Si l'on consulte la table des matières de l'année 1886 par exemple, sur 258 rubriques classées géographiquement, 26 concernent l'Afrique (dont 18 l'Afrique tropicale) et 118, soit $46 \%$, l'Etat Indépendant du Congo, chaque rubrique pouvant d'ailleurs comprendre plusieurs articles. En 1890, les proportions restent du même ordre : sur 255 rubriques, 111 pour l'Etat Indépendant du Congo (44 \%) et 88 pour le reste de l'Afrique. A la fin des années 1890, la part du Congo diminuera pour faire place aux informations sur l'Asie et tout particulièrement sur la Chine, qui sera l'autre théâtre des activités belges outre-mer. Elle ne sera plus ainsi que de $21 \%$ en 1899 . Mais la part en pages sera toujours prépondérante.

6 Le Mouvement Géographique est donc un journal qui doit inévitablement retenir l'attention de tous ceux qui s'intéressent à l'histoire de la colonisation belge au Congo et à l'histoire de sa connaissance géographique. Bien qu'il n'apporte que rarement des documents de première main, il constitue une source documentaire de grande importance. A son niveau de spécialisation et de périodicité, le Mouvement Géographique n'a pas de véritable équivalent dans les autres pays colonisateurs. Certes il y a eu ailleurs un "Journal des voyageurs" ou une "Koloniale Zeitung" mais le Mouvement Géographique apparaît comme une entreprise très originale.

Le Mouvement Géographique naît au moment où les efforts de Léopold II sont sur le point de déboucher sur la création d'une colonie mais d'une colonie d'un type un peu particulier puisqu'elle relèvera d'un individu et non d'un pays. Il est fondé quelques mois avant l'ouverture de la Conférence de Berlin où les puissances européennes vont se pencher sur le sort de l'Afrique. Son propriétaire est l'Institut National de Géographie, un "institut scientifique privé", en fait une société anonyme qui vient de patronner en Afrique l'expédition du $\mathrm{D}^{\mathrm{r}}$ Joseph Chavanne, chargé de lever la carte du Congo, de l'embouchure au Stanley Pool, et de "chercher à résoudre, dans un voyage de découvertes, le problème du lac Liba et de la rivière Ouellé"3. Le rédacteur en chef est Alphonse-Jules Wauters. Le journal se présente sous le couvert de la géographie. Son programme, exposé dans l'éditorial du premier numéro (le numéro est du 6 avril 1884, le texte est daté du 5), est de "donner utilement à un tel mouvement [de renaissance géographique], l'appui et la coopération d'un organe spécial de propagande". Le journal, qui se présente comme le "journal populaire des sciences géographiques", a choisi le format de la gazette. Il "stimulera l'esprit d'entreprise et soutiendra tous ceux qui s'efforcent d'ouvrir des horizons nouveaux, d'élargir le terrain de notre activité, de pousser le pays à sortir pacifiquement de ses étroites frontières". L'éditorial rappelle évidemment que ce mouvement de renaissance géographique est dû "en grande partie avons-nous besoin de le dire ? - à l'initiative privée du Roi, à sa philanthropie éclairée, à son action persévérante". Le Mouvement Géographique se veut un journal d'information. 
"En cherchant à réaliser ce programme, son but n'est pas seulement de servir de memorandum à l'homme d'étude, au professeur, à l'officier, au touriste; de renseigner l'homme du monde qui suit en curieux le mouvement géographique, souvent mêlé au mouvement politique; mais encore d'intéresser à la géographie la jeunesse de nos écoles et de nos universités, de contribuer au développerment de son instruction. Par ses cartes, ses illustrations, ses articles d'à-propos et d'actualité, il cherche à pénétrer dans la vie des familles: le père comme l'instituteur trouvera dans ses colonnes le moyen de rendre attrayante l'étude d'une science qui, entendue dans le sens le plus large et le plus élevé du mot, diffère essentiellement de cette nomenclature sèche et mesquine à laquelle on se bornait jadis dans nos écoles".

\section{Journal d'un mouvement colonial ou journal d'un individu?}

Il est évident qu'étant donné le moment où ce journal fait son apparition, on peut se demander s'il s'agit uniquement d'une initiative privée qui correspond a un besoin de l'époque ou d'une réponse à une sollicitation précise qui viendrait par exemple du roi ou de son entourage. Il serait intéressant aussi de savoir quelle a été la source initiale de financement. Nous n'avons pas eu la possibilité d'effectuer des recherches qui auraient éclairé ces points. Nous verrons un peu plus loin que des membres de l'entourage du roi ont joué un rôle, du moins par leurs conseils. Mais on peut considérer que ce journal, qui paraîtra tous les quinze jours, le dimanche, et qui sera complété, à partir de la fin 1891, les autres dimanches, par "Le Congo illustré, Voyages et travaux des Belges dans l'Etat indépendant du Congo", pour devenir finalement hebdomadaire, en 1896, après la fusion des deux journaux, a été largement l'oeuvre personnelle d'A.-J. Wauters (1845-1916), qui en est le rédacteur en chef puis, à partir de 1890, le directeur. Le journal ne lui survécut que de quelques années.

Alphonse-Jules Wauters est à coup sûr une personnalité originale 4 . Sans doute n'est-il pas au départ un géographe bien qu'il s'intitule ainsi. En fait il ne l'est ni plus ni moins que la plupart des membres fondateurs et des membres actifs des sociétés de géographie. Ceuxci, dans le domaine de la géographie, sont le plus souvent - l'Université ne formant pas de géographes - des autodidactes que les livres d'explorations ou de voyages ont passionnés et qui sont encadrés au point de vue scientifique, par des astronomes, des historiens, des officiers d'artillerie et quelques professeurs de l'enseignement secondaire.

A.-J. Wauters a fait, avec son frère Emile, qui fut un peintre renommé, un voyage en Egypte qui l'a beaucoup impressionné. Il a été membre de la société belge de géographie. Il en a même été le secrétaire-adjoint ${ }^{5}$. Il a rempli la même fonction au bureau du Congrès de géographie commerciale que cette société organise à Bruxelles en 1879. Il fait suivre alors son nom de la mention "négociant". En effet, fils d'un greffier à la Cour de Cassation, A.-J. Wauters, après ses études secondaires, a été installé par son père commerçant (en parapluies !) mais il a voulu entreprendre aussi une carrière littéraire, écrivant quelques pièces de théâtre, dont deux furent jouées, et le livret d'une opérette. Mais, après l'échec de son entreprise commerciale, il entre dans la carrière de journaliste en tenant la critique artistique de l'Echo du Parlement, dont son beau-père est directeur. Historien de l'art, il a écrit une Histoire de la peinture flamande, et c'est à ce titre qu'il entrera à l'Académie Royale de Belgique ; il enseignera l'histoire de l'art à l'Académie - c'est-à-dire 
l'Ecole - des Beaux-Arts de Bruxelles. Mais il s'est en même temps passionné pour les exploits des explorateurs de l'Afrique Noire. C'est donc un géographe en chambre mais il serait injuste de lui dénier la qualité de géographe. C'est un géographe engagé dans le journalisme, ou mieux peut-être, un journaliste "colonial", le "fondateur de la Presse coloniale belge", dira de lui R.J. Cornet ${ }^{6}$. Pendant trente ans, il va noter, compiler tout ce qui s'écrit et se dit sur l'Afrique Noire, mais surtout sur le Congo, porter sur des cartes les itinéraires des explorateurs, mettre régulièrement à jour une carte de l'Afrique centrale et écrire une montagne d'articles qui sont toujours d'une grande conscience scientifique. Sur ce plan, un de ses titres de gloire sera d'avoir résolu, par le raisonnement, un des derniers grands "blancs" de la carte d'Afrique, le cours inférieur de l'Uele ${ }^{7}$. Schweinfurth croyait que cette rivière allait rejoindre le lac Tchad. Wauters, après le voyage de Grenfell, qui a remonté l'Ubangi jusqu'à ses premiers rapides et constaté la courbe qu'il décrivait en amont, fait l'hypothèse, en 1885, que cette rivière est le cours inférieur de l'Uele. Il le dit dans un article au titre très journalistique qui occupe toute la largeur de la première page du numéro: "Le dernier grand blanc de la carte d'Afrique. Un nouveau Congo. Le problème de l'Ouellé. Hypothèse nouvelle". Mais son hypothèse laisse sceptiques les explorateurs. Il faudra attendre 1887 pour que le voyage de Van Gèle la confirme. Sans doute est-il un peu abusif de comparer Wauters, comme l'avait fait son ami Thys ${ }^{8}$, à l'astronome Le Verrier découvrant par le calcul l'existence de Neptune mais incontestablement, il a été, tout en n'ayant jamais mis le pied en Afrique centrale, le plus grand connaisseur de son temps de la géographie du Congo.

11 Le Mouvement Géographique publiera d'ailleurs de nombreux articles "géographiques" de la main de Wauters. Certains annonceront la solution qu'un voyage d'exploration apporte à un problème comme l'expédition Wissmann pour celui du Kasai. D'autres discuteront par exemple l'écoulement du lac Mouta Nzige (lac Edouard). Ses eaux vontelles rejoindre le lac Albert Nyanza, donc le Nil ou se dirigent-elles vers le sud c'est-à-dire en fin de compte vers le Congo ? Cette question aboutira en 1897 à un article dont le titre barre la première page: "Comment le bassin de l'ancienne mer intérieure "AlbertEdouard" a été rattaché au bassin du Nil par la Semliki "(3 janvier 1897, nº ${ }^{\circ}$. En 1894, dans deux grands articles, Wauters tentera même une synthèse générale du relief et de sa formation : "Le relief du bassin du Congo et la genèse du fleuve"9. Tout cela, malgré une terminologie parfois discutable, est du même niveau que celui des revues géographiques proprement dites. Wauters fera d'ailleurs une description géographique détaillée de l'Etat Indépendant du Congo dans un livre publié en $1899^{10}$.

12 Mais ce géographe "professionnel", comme on dirait peut-être aujourd'hui, est avant tout engagé dans l'action coloniale. Dès la conférence géographique de Bruxelles, il défend les initiatives africaines de Léopold II. Il écrit, dans le bulletin de la société belge de géographie, un article réfutant les prétentions portugaises sur l'embouchure du Congo ${ }^{11}$. Il publie aussi, à la même époque, un mémoire sur le Zambèze ${ }^{12}$. Ses contemporains ont vu, dans "Le Mouvement Géographique", un organe de presse créé à l'initiative de Léopold II pour défendre sa politique africaine. Formellement ce n'est pas exact mais il est évident qu'il était dans le mouvement de cette entreprise. Wauters s'était lié d'amitié avec un jeune officier, Albert Thys, qui était un collaborateur du colonel Strauch, à

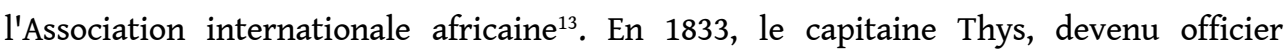
d'ordonnance du roi, est engagé davantage encore dans la politique africaine de celui-ci et aurait communiqué son enthousiasme à son ami. C'est vraisemblablement sous son influence que Wauters accepte le poste de rédacteur en chef du journal. Il est donc 
difficile de ne pas voir, dans Le Mouvement Géographique des premières années, un reflet au moins indirect de la politique congolaise du Palais de Bruxelles. C'est ainsi que l'ont compris en tout cas beaucoup de ses lecteurs et de ses détracteurs. A.-J. Wauters s'en est défendu à plusieurs reprises. Dans la "Bibliographie du Congo", qu'il publie, avec l'aide de son collaborateur Ad. Buyl, en 1895, il dit de son journal, dans la rubrique qui le concerne : "contrairement à une idée généralement accréditée, surtout à l'étranger, il n'a jamais été subsidié, ni inspiré par le gouvernement de l'Etat indépendant du Congo" ${ }^{14} \mathrm{En}$ fait, quand Wauters écrit ces lignes, le journal a pris ses distances avec la politique du souverain car il est devenu, depuis mars 1890, la propriété de la Compagnie du Congo pour le Commerce et l'Industrie. Le voilà donc en fait le journal des sociétés belges au Congo. Or celles-ci vont avoir à se plaindre des mesures prises par l'Etat indépendant.

\section{Les premières années : la communauté de vues avec l'Etat Indépendant du Congo} périodes. Il est indéniable que, jusqu'en 1890, le journal défend sans réticence les actes et les réalisations de l'Etat Indépendant du Congo et la politique coloniale de Léopold IL Un de ses biographes écrira que son activité au Mouvement Géographique valut alors à A.-J. Wauters l'amitié du roi ${ }^{15}$. Dès son deuxième numéro (20 avril 1884, p. 5), Wauters peut annoncer: "L'Afrique compte un Etat de plus", le Sénat de Washington venant de reconnaitre l'Association Internationale Africaine comme le pouvoir dominant dans le bassin du Congo. "C'est un hommage solennel rendu par les représentants d'un grand peuple, non seulement à la haute pensée qui a créé l'oeuvre, mais également à la vaillance et à l'énergie avec lesquelles elle est conduite". D'autres textes de la même veine ne laissent aucun doute sur la position du journal. Wauters, dans plusieurs éditoriaux, écrira des plaidoyers en faveur de l'Association Internationale Africaine qui veut "essayer de fonder en Afrique centrale un Etat libre, indépendant et moderne, sans douane et sans barrière... dans un but désintéressé, philanthropique et humanitaire", qui "veut ouvrir librement l'Afrique à la civilisation et au commerce de toutes les nations" (1884, $\left.\mathrm{n}^{\circ} 10\right)$. Il $\mathrm{s}^{\prime}$ efforce de réfuter les objections anglaises $\left(1884, \mathrm{n}^{\circ} 10\right)$ et les prétentions portugaises $\left(1884, n^{\circ} 13\right)$.

Pendant les deux premières années, Wauters cherche aussi à donner du Congo une image attrayante. Il emboîte le pas à Stanley qui exalte les richesses du Haut-Congo, c'est-à-dire du pays qui se trouve en amont du Stanley Pool ${ }^{16}$. Il réfute les propos de voyageurs d'Europe centrale qui décrivent le Congo sous des couleurs sombres à partir de ce qu'ils ont vu dans le Bas-Congo ${ }^{17}$. Pour Wauters, comme pour Stanley, il ne faut pas juger de l'intérieur sur ce qu'on voit près de la côte. En rapportant une entrevue avec un officier de l'Association Internationale, Van Gèle, il lance le thème des "Indes africaines", c'est-àdire d'un territoire doté de fabuleuses richesses qui ne demandent qu'à être exploitées ${ }^{18}$. L'insalubrité, qui donne à l'Afrique une réputation redoutable, est contestée ou du moins ramenée à un niveau qui ne doit plus inquiéter. Tout cela va bien dans le sens d'une promotion de l'aventure coloniale et d'un appel aux milieux d'affaires belges en faveur du Congo. Seul le thème de la population, sur lequel Stanley insistait pourtant, est négligé. Wauters y reviendra cependant en 1895, en interrogeant sur la question tous les explorateurs ( 5 articles) pour conclure enfin, dans le $n^{\circ} 8$ du 20 février 1898 (colonnes 103-106), ce qu'en fait il avait sans doute, dès le début, l'intention de démontrer, c'est-à- 
dire que l'estimation de Stanley (celle donnant 28 millions d'habitants) est, tout compte fait, la meilleure.

15 A la fin des années 1880 , le journal s'inquiète de la traite des esclaves à laquelle Wauters consacre trois gros articles, (numéros des 26 août, 9 septembre et 7 octobre 1888) et commence à aborder (numéro du 4 novembre) le problème arabe. Ces articles restent donc dans la ligne de la politique de l'Etat Indépendant et préparent en somme l'opinion publique à la Conférence de Bruxelles de 1890 qui étudiera les moyens de mettre un terme à la traite et, à l'occasion de laquelle aussi, Léopold II se fera accorder par les puissances signataires de l'Acte de Berlin l'autorisation de lever des droits d'entrée pour se procurer les ressources nécessaires au fonctionnement de l'Etat.

\section{La deuxième période : le journal des sociétés coloniales}

A partir de 1890, le Mouvement Gégraphique entre dans une nouvelle période. Il cesse d'être confondu avec un porte-parole officieux ou bénévole de l'Etat Indépendant. Depuis quelques années, il se préoccupe des sociétés belges qui sont créées pour travailler au Congo, surtout de la Compagnie du Congo pour le Commerce et l'Industrie, qui doit notamment faire l'étude du chemin de fer du Congo et dont il publie en 1887 (numéro 16) un grand placard publicitaire annonçant l'émission de 2.000 actions. Il consacre de nombreux articles à la société du chemin de fer et en 1889, au débat qui la concerne à la Chambre. Wauters conclut d'ailleurs l'article qu'il consacre à ce débat par un vibrant "En avant !"19.

En mars 1890, le Mouvement Géographique devient la propriété de la Compagnie du Congo pour le Commerce et l'Industrie, la compagnie d'Albert Thys. Il publiera régulièrement les rapports des Compagnies du Congo installées à la rue Bréderode, où sera d'ailleurs désormais son adresse. Dans un avis au lecteur, le 7 septembre 1890, le Mouvement Géographique dément officiellement être un organe de l'Etat Indépendant du Congo et se proclame "une publication indépendante, propriété de A.-J. Wauter [nous avons vu que ce n'était pas tout à fait vrai] qui, de sa propre initiative, a décidé de consacrer son journal aux affaires du Congo". Dans un placard publicitaire de sa prime de fin d'année 1891, "Le Commerce Belge au Congo", il se présente avec le sous-titre "Organe des intérêts belges au Congo". Quelques années plus tard, ce sera: "Organe des intérêts belges dans les pays d'Outre-Mer". En 1896, Wauters fait suivre son nom, dans l'en-tête du journal, de la mention "secrétaire général des Compagnies belges au Congo" puis, quelques années plus tard, "secrétaire général de la Compagnie du Chemin de fer du Congo". Le Mouvement Géographique apparaît dès lors comme le journal des compagnies coloniales du groupe Thys, ou comme on dit aussi à l'époque, de la rue Bréderode. Il offrira même à ses abonnés, en 1896, un droit de préférence pour les nouvelles actions que la Compagnie du chemin de fer du Congo vient d'émettre et qui font partie du portefeuille de la Compagnie du Congo pour le Commerce et l'Industrie. Dans l'éditorial de son dernier numéro, le 31 décembre 1922, le Mouvement Géographique reconnaîtra sans détour que "le général Thys... fit assurer l'existence matérielle du journal par la compagnie du Congo pour le Commerce et l'Industrie qu'il dirigeait et dont il était l'âme. "Mais le journal précise: "Il [le général Thys] ne demanda à A.-J. Wauters et à ses colaborateurs rien d'autre que de poursuivre l'oeuvre qu'ils avaient commencée sans en 
changer l'esprit". Et le journal ajoute que "ses inspirateurs ne furent jamais les maîtres de l'heure. Ils s'appelèrent Banning, Beernaert, Paul Janson, Strauch, Van Neuss, pour ne citer que ceux, parmi les grands citoyens, qui ont achevé leur tâche en ce monde".

Le Mouvement Géographique va consacrer une part importante de son contenu aux activités des sociétés commerciales de la rue Bréderode. La création de la Compagnie du Katanga et les expéditions menées dans ce territoire nourrissent des numéros entiers. Le journal publie systématiquement les rapports annuels de ces sociétés et plus particulièrement celui de leur société-mère. Il n'est pas étonnant de lui voir consacrer un nombre élevé de rubriques au chemin de fer du Congo dont il suit la progression kilomètre par kilomètre (44 rubriques en 1898, l'année du triomphe quand le 3 juillet, le rail est officiellement inauguré ; ce dimanche-là Wauters intitule son article "La conquête du Congo").

Le Mouvement Géographique, qui est devenu la propriété des sociétés coloniales, prend bientôt ses distances envers l'Etat Indépendant du Congo. Le roi s'est engagé en effet dans sa politique du domaine qui tend à réserver à l'Etat ou à de grandes compagnies auxquelles il accorde un monopole, l'exploitation des terres qu'il considère comme vacantes. Cela ne va pas sans gêner les compagnies de la rue Bréderode. Certes le journal ne réagit pas lorsque la Compagnie du Katanga, filiale de la Compagnie du Congo, est la première bénéficiaire de ce monopole. Mais en 1892, le Mouvement Géographique change brusquement de ton. Les autorités de l'Etat Indépendant viennent d'interdire aux indigènes de vendre du caoutchouc et de l'ivoire dans les rivières Ubangi, Uellé, Bomu et leurs affluents en raison des droits de l'Etat sur les domaines et menacent de sanctions les compagnies commerciales qui passeraient outre à cette interdiction. L'affaire concerne la Société Belge du Haut Congo, filiale de la Compagnie du Congo. Elle porte atteinte directement et de façon précise à la liberté commerciale qui avait été garantie dans le bassin du Congo. Wauters est accablé sinon indigné. "Pour ce qui nous concerne personnellement, écrit-i ${ }^{20}$, nous qui signons ces lignes et qui, depuis quinze ans, servons sans marchander et avec foi l'idée généreuse et pure, créatrice de l'oeuvre, nous ne cacherons pas que nous sommes déconcerté et troublé". Le ton monte. Quinze jours plus tard, Wauters parle "d'un triste spectacle. Un fatal moment d'arrêt, de recul se produit dans l'édification de la magnifique oeuvre coloniale que la Belgique poursuit au Congo". Il veut croire qu'on a trahi la pensée royale "en l'interprétant comme on vient de le faire en Ubangi".

Le Mouvement Géographique consacrera entre la fin juillet et le mois de décembre 1892 pas moins de 49 articles, notes et rubriques au "conflit entre l'Etat Indépendant du Congo et les sociétés commerciales". Il dénonce de plus en plus vivement les dangers du système que l'on veut mettre en place car l'Etat va ériger "ses agents en concurrents, en les armant de tous les pouvoirs militaires et judiciaires". Il y voit "matière à mille conflits, à des abus de toute nature". Il estime que "l'Etat trafiquant et ayant à sa solde des agents excités à supprimer la concurrence voisine, c'est là une situation d'une gravité exceptionnelle"21. Il s'écrie même : "la mesure est comble"22. Il rappellera, dans un titre à la une, "les déclarations de la Conférence de Berlin en faveur de la liberté commerciale au Congo" (11 septembre 1892, n 21 ). Il fera des conférences sur ce sujet par exemple à la Maison du Peuple pour les socialistes belges qui veulent des informations sur le Congo. On sait qu'à ce moment un arrangement interviendra entre l'Etat et les compagnies commerciales concernées. Le roi leur abandonna, dira Wauters par la suite, un os à ronger ${ }^{23}$. Mais cet incident a fait de Wauters un ardent partisan de la reprise, la plus prompte 
possible, du Congo par la Belgique. La Compagnie du Congo pour le Commerce et l'Industrie, de son côté, chaque année, va l'appeler de ses voeux dans son rapport. Notant, en 1895, dans l'introduction à sa "Bibliographie du Congo", que l'impression de celle-ci a commencé au moment où était déposé à la Chambre un projet de cession de l'Etat Indépendant à la Belgique et constatant que ce projet avait été suspendu, Wauters écrit : "Nous avons cependant la conviction de ne pas avoir travaillé en vain, même au point de vue national. Nous nous refusons en effet à admettre comme une impossibilité morale que les Belges renoncent finalement à présider aux destinées du grand fleuve africain"24 .

L'évolution de Wauters à cette époque est parallèle à celle d'Emile Banning, ce directeur aux Affaires Etrangères qui fut longtemps le conseiller du roi mais qui n'avait lui aucun lien avec les sociétés coloniales. Banning, qui avait préparé et participé aux négociations qui avaient permis à l'Etat Indépendant de créer son système douanier, s'était détaché des vues du roi. "Depuis le mois de mai 1891, la politique de l'Etat du Congo n'a plus fait que dévier... le Roi a répudié progressivement les principes de la science économique pour s'engager dans des voies rétrogrades. Pour subvenir à des expéditions coûteuses et inutiles, la fiscalité a été poussée à outrance. De 1890 à 1892, j'eus plus d'une lutte pénible à soutenir contre lui sur ce terrain. L'invention de la théorie du domaine qui allait droit à la création d'un vaste monopole d'Etat, fut le coup de grâce: c'était l'expulsion du commerce libre, la ruine des Compagnies belges, le dos tourné à l'Acte de Berlin"25. La rédaction d'un mémoire achevé en octobre 1892 sur "La liberté commerciale dans le bassin conventionnel du Congo d'après l'acte général de Berlin" consacra la rupture définitive avec le roi. En 1894, Banning réclama même la reprise du Congo par la Belgique comme une mesure de salut public ${ }^{26}$.

\section{La rupture définitive avec l'Etat léopoldien}

Le divorce entre le Mouvement Géographique et la politique africaine du roi, qui avait commencé à se produire pendant les années 1890, atteindra un autre point culminant pendant l'Affaire congolaise en 1903. Certes le journal ne lance pas d'attaques violentes mais tous les débats parlementaires, tous les points de vue hostiles exprimés en Belgique comme à l'étranger sont reproduits in extenso et, quand il y a défense de l'Etat, celle-ci est très molle. Ainsi en 1901, il publie sans commentaire le texte de la pétition de "La société anglaise pour la protection des indigènes au Congo" qui dénonce des actes de violence et des atrocités. Il le fait suivre de la réponse de "L'Etoile belge", qui, dit-il, "passe pour être le journal officieux de l'Etat indépendant du Congo" et qui n'y voit qu'un tissu de calomnies ${ }^{27}$. En tout cas, il ne rejette pas comme des insultes les affirmations des mouvements anglais contrairement à ce que fait au même moment, par exemple, le bulletin de la Société royale de géographie d'Anvers. Dans une lettre au journal du 2 mars 1908, donc quelques années plus tard, E. Morel signalera que le Mouvement Géographique a été une des rares exceptions parmi les journaux belges à n'avoir pas couvert la Congo Reform Association d'injures et de mépris ${ }^{28}$. Le Mouvement ne cessera de proclamer la nécessité d'une reprise rapide du Congo par la Belgique. En 1901, d'ailleurs, alors que la question congolaise était l'objet d'interventions à la Chambre et deux ans avant le point culminant de la crise, l'éditorial du 5 mai $\left(n^{\circ} 18\right)$ s'intitulait "Pour l'annexion immédiate du Congo".

R. Cambier, qui a écrit la notice sur Wauters dans la Biographie Coloniale, dit de façon un peu pincée: "Alphonse Wauters, quoique colonial au fond de l'âme et artisan de la 
première heure de notre grande oeuvre africaine, eut le tort d'intervenir avec trop de vivacité au moment même où, critiquant l'attitude du Souverain, on risquait de faire passer en d'autres mains la mission civilisatrice que nous avons accomplie depuis sur le Continent Noir". Cambier, rappelant que Wauters avait accepté des fonctions dans un groupe de sociétés coloniales, ajoute: "Sans qu'il soit question pour les gens avertis de mettre en doute sa profonde loyauté, il est certain qu'une telle situation était de nature à diminuer, dans l'esprit du grand public, la force convaincante de ses appréciations" 29 . Léopold II d'ailleurs ne les estimait guère écrivant même à son secrétaire, le baron Carton de Wiart, que, lorsqu'il rencontrera le colonel Thys, de lui dire nettement "de conseiller à son employé Wauters de surveiller son langage. Si le Roi apprenait que Wauters, qui dépend absolument de Thys, continue à manquer de réserve, les relations de S.M. et du Colonel s'en ressentiraient" ${ }^{130}$. Ce n'est cependant pas dans le Mouvement Géographique que l'on trouve les propos dont se plaint le roi. Le ton y reste modéré, plus même qu'en 1892. Mais il est vraisemblable que, dans ses conférences et ses propos publics, Wauters se soit montré plus incisif. Un de ses biographes le décrit en effet comme un "conférencier vivant et convaincu"131.

La position de Wauters est dans la suite logique de l'attitude qu'il avait adoptée dès 1892. Elisée Reclus avait lui aussi dit des choses assez semblables dans sa Nouvelle Géographie Universelle où il redoutait déjà de voir de grands domaines se constituer ${ }^{32}$. Dans "L'Homme et la Terre", il constate que ses craintes se sont confirmées: "De tous les méfaits perpétrés en Afrique par les blancs, ceux qui, depuis vingt ans, ont été commis dans l' "Etat Indépendant du Congo" sont peut-être les plus horribles : ils sont les plus récents. Mais quel est l'Anglais, l'Allemand, le Français dont la main est assez pure pour que sa protestation ne soit entachée de partialité" 33 . Il n'en accable pas d'ailleurs la Belgique qui se trouve "associée malgré elle à la politique de l'Etat Indépendant du Congo" et que "le caoutchouc rouge - rouge du sang de l'indigène "vient de brouiller avec l'Angleterre $^{34}$.

Wauters affirmera avoir vu, le premier, les dangers de la politique suivie par Léopold II. Ecrivant en 1910 à Morel, qui avait dirigé, en Angleterre, la campagne contre les "atrocités congolaises" et la politique de l'Etat Indépendant, il lui dit: "Quel cauchemar que ce Congo léopoldien et combien j'ai dû ronger mon frein !... Personne plus que moi en Belgique n'apprécie ce que vous avez fait en Angleterre, dans ce but, en faisant éclater la lumière et la vérité. Mais je revendique [les deux mots sont soulignés dans la lettre] l'honneur d'avoir été le premier, le tout premier, à tirer l'épée et à engager le combat. Lorsqu'aujourd'hui, je jette un coup d'oeil sur le passé et l'état des esprits en 1892, je demeure, vous le dirais-je, étonné de mon audace et de mon courage" ${ }^{135}$. Sans doute Wauters exagère-t-il quelque peu mais c'est incontestablement son honneur d'avoir été le seul "géographe" belge à avoir éprouvé et surtout à avoir exprimé (en dehors du cas de Reclus, qui, bien qu'en Belgique, n'appartenait pas au groupe des géographes belges) ses doutes et ses inquiétudes, sinon ses indignations, sur la façon dont évoluaient les choses dans le Congo léopoldien. Pourtant il ne faut pas se méprendre. Wauters n'est pas animé seulement de sentiments humanitaires. Il ne remet pas en cause le système colonial. Pour lui, les violences et les exactions n'en sont pas le produit, ni même des bavures. Elles proviennent du système de monopole mis en place par l'Etat Indépendant. C'est une position d'ailleurs très voisine de celle de Morel et de son Congo Reform Movement en Angleterre. 
En 1908, le Mouvement Géographique attaque encore de façon plus explicite la politique de Léopold II, particulièrement en ce qui concerne le domaine de la Couronne dont la création lui parait avoir été faite au détriment de sociétés commerciales déjà installées. C'est qu'il s'impatiente devant les atermoiements dans le processus de reprise du Congo par la Belgique. "Il y a treize ans qu'un premier projet d'annexion a été déposé au Parlement... Il y a trois ans que le Parlement belge a émis son premier vote de principe en faveur de la reprise... Catilina est à nos portes et nous délibérons toujours !"36.

\section{Conclusion. Le Mouvement Géographique, un témoin ou un acteur?}

Le Mouvement Géographique est un document essentiel sur les trente premières années de la Belgique au Congo. On peut y suivre presque au jour le jour la création des postes administratifs et commerciaux, les mouvements des hommes entre l'Europe et l'Afrique, les progrès des sociétés commerciales et des productions. On y trouve rassemblées des données éparses dans les différentes publications de l'époque. A ce point de vue, le Mouvement Géographique a répondu à un des objectifs officiels de ses fondateurs. L'éditorial de son dernier numéro, du dernier dimanche de 1922, dit fièrement: "Ses collections, conservées dans les grandes bibliothèques des deux mondes, resteront, en ce qui concerne le bassin du Congo et son histoire, la source de renseignements la plus complète et la plus sûre". Certes la plupart de ces informations ne sont pas originales. On n'y trouve pas d'indications sur le dessous des cartes, pas de révélations fracassantes. Il serait sans cloute malaisé d'écrire une histoire coloniale de la Belgique en ne recourant qu'à ce journal car on n'y voit pas les hommes politiques à l'oeuvre. On n'y trouve pas exposés non plus de façon systématique les principes d'une politique coloniale. On n'y trouve aussi que fort peu de renseignements sur les relations entre colonisés et colonisateurs. On y parle du Congo mais assez peu des Congolais. Les rubriques "ethnographiques" par exemple sont très peu nombreuses alors qu'elles seront plus développées dans les revues des sociétés de géographie (en fait surtout à partir du début de ce siècle).

Quelques documents iconographiques seulement concernent les peuples du bassin $\mathrm{du}$ Congo; la plupart montrent plutôt les réalisations coloniales (photos de postes, de factoreries, de voies ferrées, de chantiers, de ponts ferroviaires, de ports, d'hôpitaux, etc.). Les institutions traditionnelles sont rarement abordées. Par contre on trouve sous la plume de Wauters, notamment à la suite de débats qui eurent lieu à la Maison du Peuple sur la question du Congo, quelques notes réfutant les opinions racistes sur les Noirs africains. "Rien ne justifie l'hypothèse, écrit-il en 1892, de l'infériorité native de la race africaine. Celle-ci est douée de toutes les facilités et de tous les attributs qui permettent à une race de se développer, de s'améliorer et de s'affirmer" ${ }^{137}$. Mais bien sûr on peut considérer que de tels propos s'inscrivent aussi dans la logique d'une promotion des compagnies coloniales puisqu'ils indiquent que celles-ci sont assurées de trouver une main-d'oeuvre convenable et perfectible. On traite la question des maladies dans la mesure où celles-ci menacent la vie et l'activité des Européens. On aurait pu s'attendre à ce que le journal traduisît l'émotion qu'aurait dû susciter la tragédie de la maladie du sommeil. Sauf pour la trypanosomiase qui frappe le bétail (donc l'élevage, c'est-à-dire une activité qui peut concerner les sociétés coloniales), jusqu'en 1908, il ne lui consacre que des échos de quelques lignes. 
Il serait très intéressant de procéder, ce que nous n'avons pu faire, à une étude exhaustive et systématique du contenu du Mouvement Géographique. Elle permettrait de mieux comprendre comment certains milieux d'affaires belges ont conçu l'exploitation du Congo et comment peu à peu s'est affirmée l'idée que la Belgique pouvait devenir une puissance coloniale ${ }^{38}$. Mais il ne faut pas exagérer le rôle que le journal a pu avoir dans la marche des événements. Il nous paraît difficile en effet de soutenir que, "si le Mouvement Géographique n'avait pas existé, le cours de bien des événements aurait été différent" ${ }^{39}$.

\section{NOTES}

1. Nous avons consacré à ce thème une communication (à paraître) : "Les géographes belges et les débuts du Congo" dans le cadre du Colloque "Géographies. Colonisations. Décolonisations", organisé par le CEGET-CNRS à Bordeaux en mars 1992.

2. H. NICOLAI (1992), article cité.

3. Le Mouvement Géographique (celui-ci sera désigné dans les références suivantes sous l'abréviation M.G.), 6 avril , nº 1, p. 2.. Le M.G. sera publié de 1884 à 1922, avec une interruption due à la guerre 1914-1918. Nous ne l'étudierons que pendant la période qui correspond à l'existence de l'Etat Indépendant du Congo, c'est-à-dire jusqu'en 1908, date de la reprise du Congo par la Belgique.

4. Son prénom double le distingue de son oncle, Alphonse Wauters, archiviste de la ville de Bruxelles, qui a été lui aussi historien de l'art, qui se présente également dans certains de ses travaux comme géographe et qui a été président de la Société belge de géographie. On trouvera des indications biographiques sur A.-J. Wauters dans :

- L. Solvay (1938), Wauters (Alphonse-Jules), in Biographie Nationale, tome 27, col. 115-119.

- R. Cambier (1951), Wauters (Alphonse-Jules), in Biographie coloniale belge, 2, col. 970-972.

- R. J. Cornet (1949), Le fondateur de la presse coloniale. A.J. Wauters, Revue Coloniale Belge, $1^{\mathrm{er}}$ décembre 1949, pp. 780-782.

5. Il semble que Wauters n'ait plus eu d'activité à la Société belge de géographie à partir du moment où il s'occupe du Mouvement. Son nom n'apparaît plus dans la liste des membres. Wauters aura même une altercation dans son journal avec le secrétaire général de la société Jean Du Fief, les deux personnages se reprochant mutuellement de puiser des données dans la revue de l'autre sans citer leur source (M.G., juillet 1887, $\mathrm{n}^{\circ} 16$ ).

6. R.J. Cornet (1949), article cité.

7. M.G., 31 mai $1885, \mathrm{n}^{\circ} 11$, pp. 41-44.

8. L. Solvay (1938), article cité, col. 118 et R. Cambier (1951), article cité, col. 970.

9. M.G., 1897, $\mathrm{n}^{\circ} 2$ (10 janvier), col. 13-18.

10. A.-J. Wauters (1899), l'Etat Indépendant du Congo, Falk, Bruxelles.

11. A.-J. Wauters (1883), Le Congo et les Portugais. Réponse au Mémorandum publié par la Société de Géographie de Lisbonne, Bulletin de la société belge de géographie, pp. 234-278.

12. A.-J. Wauters (1878 et 1879), Le Zambèze. Son histoire, son cours, son bassin, ses produits, son avenir, Bulletin de la société belge de géographie, $2^{\text {e }}$ année, pp. 8-34, 114-138, 383-405, 566-621 et $3^{\mathrm{e}}$ année, pp. 450-482.

13. R.J. Cornet (1949), article cité.

14. A.-J. Wauters (1895), Bibliographie du Congo, Bruxelles, p. 2. 
15. L. Solvay (1938), article cité, col. 117.

16. Nous avons traité ce thème dans $\mathrm{H}$. Nicolaï (1988). L'image de l'Afrique centrale au moment de la création de l'Etat Indépendant du Congo in Recueil d'études "Le centenaire de l'Etat Indépendant du Congo", Académie royale des Sciences d'Outre-Mer, Bruxelles, pp. 13-39.

17. On trouvera un bel exemple de ce type de réfutation dans le $n^{\circ} 2$ du 24 janvier 1896 (pp. 5-6) où Wauters, sous le titre "L'oeuvre du Congo et M. Peschuel-Loesche" reprend, dans une colonne, les affirmations de Peschuel-Loesche et, dans la colonne voisine, des textes d'explorateurs qui, selon lui, les démentent.

18. A.-J. Wauters (1885), Les Indes Africaines. Renseignements recueillis dans une entrevue avec le Lieutenant Van Gèle, M.G., n 12, 14 juin, pp. 47-48.

19. A.-J. Wauters (1889), Le chemin de fer du Congo à la Chambre des Représentants, M.G., $\mathrm{n}^{\circ} 16$, 28 juillet.

20. A.-J. Wauters (1892), Le commerce belge au Congo, M.G., $n^{\circ} 15,24$ juillet, pp. 61-62.

21. M.G., 31 juillet 1892, $\mathrm{n}^{\circ} 16$, p. 67.

22. M.G., 7 août 1892, n' 17, p. 70.

23. A.-J. Wauters (1910), L'oeuvre congolaise. Les débuts de l'absolutisme, 1901-1904, M.G., 16 janvier, col. 32.

24. A.-J. Wauters (1895), Bibliographie du Congo, ouvrage cité, p. 22.

25. J. Stengers (1955), Textes inédits d'Emile Banning, Bruxelles, Académie royale des Sciences coloniales, p. 47.

26. Id., p. 50.

27. M.G., 21 mai 1901, $\mathrm{n}^{\circ}$ 19, La société anglaise pour la protection des indigènes au Congo, col. 223-224.

28. M.G., 1908, col. 148.

29. R. Cambier (1951) article cité, col. 971-972.

30. Baron Carton de Wiart (1944), Léopold II, Souvenirs des dernières années, 1901-1909, Bruxelles, p. 83.

31. L. Solvay (1938), article cité, col. 119.

32. E. Reclus (1888), Nouvelle Géographie Universelle, tome XIII, p. 399.

33. E. Reclus (1905-1908), L'Homme et la Terre, Librairie Universelle, Paris, tome V, p. 447.

34. Id., tome VI, p. 252.

35. Nous devons ce texte à notre collègue J. Stengers qui nous l'a aimablement communiqué : lettre de A.-J. Wauters du 5 septembre 1910 (sur papier à lettre du Mouvement Géographique), dans Papiers Morel à Londres, liasse n 8, Belgian Correspondence, 1909-1910. Un extrait de cette lettre est reproduit dans Wm R. Louis et J. Stengers (1969), E.D. Morel's History of the Congo Reform Movement, Oxford, p. 250 (dans le chapitre de J. Stengers, Morel and Belgium).

36. A.-J. Wauters (1908), L'Etat du Congo et l'Angleterre, etc., M.G., $1^{\mathrm{er}}$ mars, col. 109-131.

37. Le Congo à la Maison du Peuple, Conclusion par A.-J. Wauters, M.G., 6 mars 1892, n 5, p. 19c.

38. Il faudrait aussi rechercher quels étaient les lecteurs. Dans quelle mesure le journal débordait-il le milieu directement intéressé à l'activité coloniale ? Le journal est-il entré dans les familles bourgeoises comme l'espéraient ses fondateurs? A-t-il joué un rôle de même nature que celui que continuent à jouer des "magazines" géographiques qui, paraît-il, seraient aujourd'hui, en France, les périodiques les plus achetés par les "cadres"? Le Mouvement a-t-il vécu uniquement grâce au soutien financier de la Compagnie du Congo, c'est-à-dire d'Albert Thys, comme le laisse peut-être entendre l'éditorial du dernier numéro? Quand il a été repris par la Compagnie du Congo, en 1890, le rapport de cette société, cette année-là, le présenta comme une affaire légèrement déficitaire mais qui aurait dû, les années suivantes, dégager un léger bénéfice (M.G., 21 décembre 1890, n²9).

39. Comme l'affirme R.-J. Cornet, 1949, article cité, p. 781. 


\section{RÉSUMÉS}

The first edition of the 'Mouvement Géographique' was published in Brussels on April $6^{\text {th }} 1884$. Its primary aim was the promotion of Belgian colonisation in Africa. Although rarely publishing first hand information, the paper, nevertheless, constitutes a documentary source of great importance. Two periods can be distinguished in the history of the 'Mouvement Géographique'. Up to 1890 , the paper is wholeheartedly behind the actions and plans of the Congo Free State and the colonial policy of Léopold II. From 1890, when the paper became the property of the 'Compagnies du Congo pour le Commerce et l'Industrie', it distances itself from the Congo Free State. Thus, in analysing the editorial contents of the paper and the personality of its founder, A.J. Wauters, it becomes clear that this paper represents an essential document on the first thirty years of Belgium involvement in the Congo. Furthermore, its level of specialisation and regularity of publication means that there is no real equivalent in any other colonising country.

\section{AUTEUR}

\section{HENRI NICOLAI}

Laboratoire de Géographie Humaine - Université Libre de Bruxelles - Belgique 